\title{
Novel Approach to Linear Accelerator Superconducting Magnet System
}

\author{
Vladimir Kashikhin
}

\begin{abstract}
Superconducting Linear Accelerators include a superconducting magnet system for particle beam transportation that provides the beam focusing and steering. This system consists of a large number of quadrupole magnets and dipole correctors mounted inside or between cryomodules with SCRF cavities. Each magnet has current leads and powered from its own power supply. The paper proposes a novel approach to magnet powering based on using superconducting persistent current switches. A group of magnets is powered from the same power supply through the common, for the group of cryomodules, electrical bus and pair of current leads. Superconducting switches direct the current to the chosen magnet and close the circuit providing the magnet operation in a persistent current mode. Two persistent current switches were fabricated and tested. In the paper also presented the results of magnetic field simulations, decay time constants analysis, and a way of improving quadrupole magnetic center stability. Such approach substantially reduces the magnet system cost and increases the reliability.
\end{abstract}

Index Terms - Linear Accelerator, Superconducting magnet, Magnetic field, Superconducting Switches, Correction Coils.

\section{INTRODUCTION}

$\mathrm{T}$ HERE are several Linear Accelerator projects: ILC [1], XFEL [2], NML [3], and Project-X [4] under design and construction now. All of them use superconducting magnets to transport the beam along the whole accelerator length. Quadrupoles are used for the beam focusing and the dipole correctors are used for the beam steering. Various magnet prototypes were built and successfully tested [5-10]. The main problem for the ILC type machine is to reach several microns level of magnetic center stability. This is the most critical parameter for the long electron beam Linacs [8-9]. For proton machines [3-4] this parameter is not so critical. Another issue is that all magnets should be powered separately having large number of current leads, power supplies, and long external cables. It will be quite expensive for hundreds of magnets. The paper proposes and discusses a novel approach to take an extra advantage from the superconductivity by using the magnet ability to work in a persistent current mode. This technique is widely used in NMR superconducting magnet systems.

Manuscript received 12 September 2011.

V. S. Kashikhin is with the Fermi National Accelerator Laboratory, Batavia, IL 60510 USA (corresponding author phone: 630-840-2899; fax: 630-840-6766; e-mail: kash@fnal.gov).

\section{SuPERCONDUCTING MAGNET System}

Linear accelerators have a large number of superconducting magnets to provide the precise beam transport. Because the energy of accelerated particles increases along the accelerator length, the strength of magnets increases proportionally. Nearly all magnets in such case should be powered separately from separate power supplies. Each magnet has at least a pair of current leads, power supply, long cables to connect them, quench detection and protection systems. Such large number of elements substantially increases the system cost and reduce the magnet system reliability (See Fig. 1).

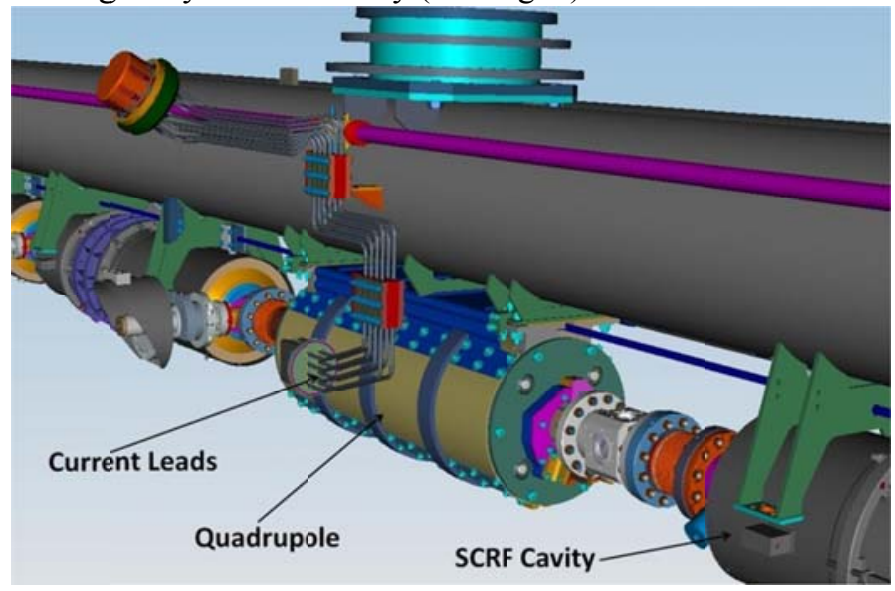

Fig. 1. Quadrupole view inside SCRF Cryomodule.

Another approach is to use the possibility of superconducting magnets to work in the persistent current mode. MRI Solenoids routinely use this technique. The main magnet system parameters should be in an agreement with the following:

- large magnet inductances;

- very low splice resistances;

- high performance persistent current switches;

- long low inductive superconducting busses;

- efficient control system.

\section{A. Electrical Schematic and Operation}

To explore the proposed approach all magnets should be combined in magnet groups having the same electrical current supply bus. It is more convenient to have two busses to power quadrupoles and dipoles separately. The electrical schematic for the single cell with the focusing, defocusing quadrupoles and vertical, horizontal dipole correctors is shown in Fig. 2. 


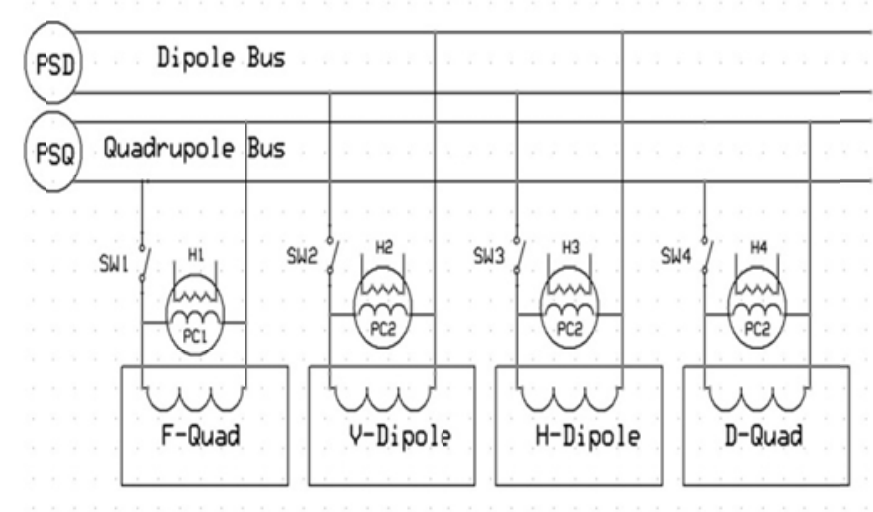

Fig. 2. The magnet system cell schematic. SWn- superconducting switch, PCn-persistant current switch, Hn - PCn heaters, PSD and PSQ dipole and quadrupole power supplies.

The number of cells powered from the single power supply is defined by the operation scenario. There are two main modes of operation: short term with a test beam, and the long term normal accelerator operation. In the first mode the magnets should be powered and their current should be changed as fast as possible. In the long term operation, the need for the magnet strength corrections is relatively rare.

Initially all switches are open and there is no current in the magnets. When all cryomodules and magnets cooled down to the operating temperature, the first magnet in the cell is powered from the corresponding power supply. In this case, the SW1 switch closed and all other switches in the cell are open. When the current reaches the specified for this magnet value, the persistent current switch is closed and the current continuously circulates through the magnet. After that, the switch SW1 must be open and the magnet electrically disconnected from the supply bus. This procedure is repeated for all quadrupoles and dipoles. Finally all magnets work in the persistent current mode and disconnected from the buses.

During acceleration and magnets tuning a test beam is used. If the Beam Based Alignment (BBA) technique is applied, the strength of each quadrupole is changed by $-20 \%$ and the signal from the beam position monitor is used to change the dipole corrector strength in a way to move the quadrupole magnetic center close the beam center. In this case, corresponding magnets are again connected to the bus by SWn switches, power supplies voltages are raised to the needed values, and persistent current switches are opened by heaters. When BBA for the quadrupole is finished, the magnet is again transferred to the persistent current mode. Electrical switches SWn are better to be superconducting or with a very low resistance. These switches are connected to superconducting busses and should have quench stoppers to intercept the heat from the switch or should be used a combination of a mechanical and a persistent current switch [16].

\section{B. Persistent Current Mode of Operation}

The key of proposed approach is the magnet operation in the persistent current mode. Parameters of the built and tested quadrupole magnet for Linear Collider is shown in Table 1.
TABLE I QUADRUPOLE MODEL PARAMETERS

\begin{tabular}{lcc}
\hline \multicolumn{1}{c}{ Parameter } & Unit & Value \\
\hline Peak operating quadrupole and dipole current & $\mathrm{A}$ & 100 \\
Magnet total length & $\mathrm{mm}$ & 680 \\
SC wire diameter & $\mathrm{mm}$ & 0.5 \\
NbTi filament size (vendor value) & $\mu \mathrm{m}$ & 3.7 \\
Cu:SC volume ratio & & 1.5 \\
Superconductor critical current at $5 \mathrm{~T}$ and $4.2 \mathrm{~K}$ & $\mathrm{~A}$ & 200 \\
Coil maximum field & $\mathrm{T}$ & 3.3 \\
Magnetic field stored energy & $\mathrm{kJ}$ & 40 \\
Quadrupole inductance at $12 \mathrm{~Hz}$ and $20^{\circ} \mathrm{C}$ & $\mathrm{H}$ & 3.9 \\
Quadrupole coil number of turns $/$ pole & & 900 \\
Yoke outer diameter & $\mathrm{mm}$ & 280 \\
\hline \hline
\end{tabular}

The magnet coils are wound from the single strand $\mathrm{NbTi}$ superconductor. The magnet winding has only 5 external splices which could be made with a very low resistance in the range of $10 \mathrm{n} \Omega$. If the magnet will operate in the persistent current mode, the current decay time constant will be in the range of 12 years for the $3.9 \mathrm{H}$ winding inductance and $10 \mathrm{n} \Omega$ total external circuit resistance. The magnet current will decay with the rate $0.02 \%$ /day. It allows a very stable magnet operation free from the power supply noise produced by the commonly used low cost switching power supplies.

\section{Persistent Current Switch}

Various configurations of the persistent current switches were built and tested [10-13]. Most of them are thermally controlled by heaters. The two switches shown in Fig. 3 were designed and built at Fermilab. The switch has two bifilar wound coils: superconducting and heating.

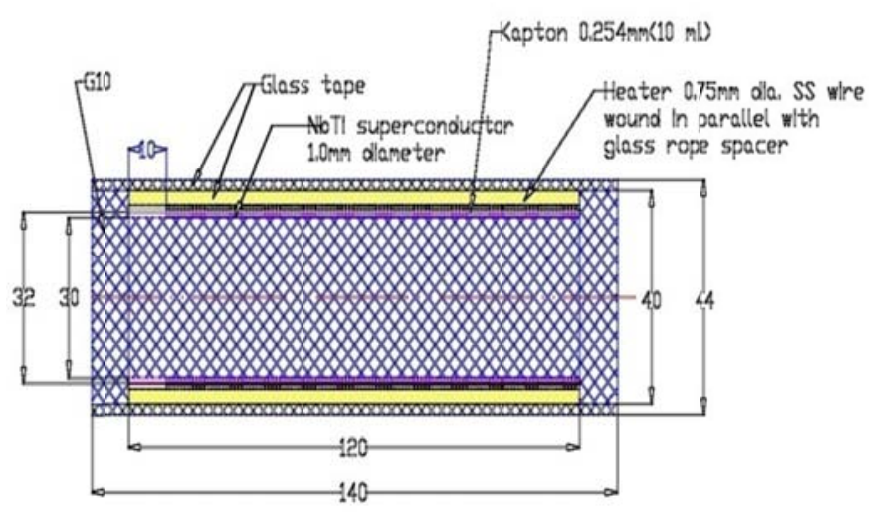

Fig. 3. Persistent current switch cross-section. Dimensions are in $\mathrm{mm}$.

D. Turrioni from FNAL successfully tested these switches [14]. During the test, no quenches were observed up to $150 \mathrm{~A}$ current. The switch parameters are shown in Table 2.

Thus, the persistent current switch is capable to open or close the magnet circuit in the range of 2-5 s. During the magnet quench or when the switch is open, most of the magnet energy will be dissipated in the switch, which, in this case, is working as an external dump resistor. The voltage at 
100 A current will be limited to $320 \mathrm{~V}$ by the open switch resistance of $3.2 \Omega$, which is acceptable.

\section{TABLE II SWITCH PARAMETERS}

\begin{tabular}{lcc}
\hline \multicolumn{1}{c}{ Parameter } & Unit & Value \\
\hline Peak operating current & $\mathrm{A}$ & 150 \\
SC coil resistance at $20^{\circ} \mathrm{C}$ & $\Omega$ & 7.8 \\
Heater resistance & $\Omega$ & 23.5 \\
NbTi wire diameter & $\mathrm{mm}$ & 1.0 \\
Superconductor stabilization material & & $\mathrm{CuNi}$ \\
$\begin{array}{l}\text { Stainless steel heater wire diameter } \\
\text { Heater current }\end{array}$ & $\mathrm{mm}$ & 0.75 \\
$\begin{array}{l}\text { Switch performance at } 100 \text { A SC current, and } \\
\text { (0.5 A, 3 s) heater current and time: }\end{array}$ & $\mathrm{A}$ & 0.5 \\
$\quad-\quad \begin{array}{l}\text { Transition from the superconducting to } \\
\text { the normal condition }\end{array}$ & $\mathrm{s}$ & 1.8 \\
$\quad \quad \quad \begin{array}{l}\text { Transition from the normal to the } \\
\text { superconducting condition }\end{array}$ & $\mathrm{s}$ & 4.3 \\
$\begin{array}{l}\text { Switch open resistance at 0.5 A, 3 s) heater } \\
\text { current and time }\end{array}$ & $\Omega$ & 3.2 \\
\hline \hline
\end{tabular}

Nevertheless, to eliminate the quench detection system it is better to use the passive protection circuit connected in parallel with the switch. This circuit consists of cold diodes and shunt resistor as shown in Fig. 4.

\section{Quadrupole MAgnetic CENTER Stabilization}

One is the most complicated problem with the quadrupole magnets for Linear Colliders is the magnetic center stabilization [5-10]. It may be achieved with a magnet having:

- Very small superconductor magnetization effects by using several microns filament size;

- Mechanically very stable cold mass, and support structure;

- Very low field distortions under Lorentz forces.

Another way is to use superconducting stabilization coils. Because the quadrupole magnetic center shift is defined by the dipole field component, stabilization coils should have dipole configuration (See Fig. 5).

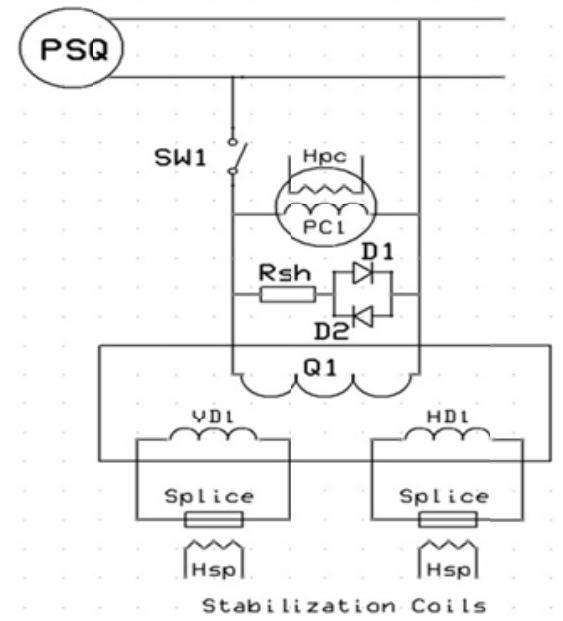

Fig. 4. Quadrupole magnet schematic. Q1 - quadrupole winding, Dn - cold diodes, Rsh - protection shunt resistor, VD1 - vertical dipole, HD1 horizontal dipole.
During the magnet operation and BBA in Linacs, these coils should be short circuited. In this case, any dipole field component change will be eliminated by the current induced in this coil. The stabilization coil inductance should be relatively large and the splice resistance very low to obtain a reasonably long decay of the induced current. The induced currents will be low because in the ideal geometry there is no coupling between quadrupole and dipole windings. Only a misalignment between quadrupole and dipole fields will cause the dipole current. In this case, instead of the persistent current switch there may be used the coil external splice area combined with a spot heater (See Fig. 2) to transfer the coil in the normal condition to clear the induced currents when needed.

\section{A. Simulation of the Stabilization Coil}

The proposed approach was investigated by using ELEKTRA OPERA3d code [16]. The model geometry is shown in Fig. 5. This is an air core magnet variant. The shell type dipole coil was shifted $1 \mathrm{~mm}$ in the X-direction to check the quadrupole magnetic center fluctuations at different currents shown in Fig. 6.

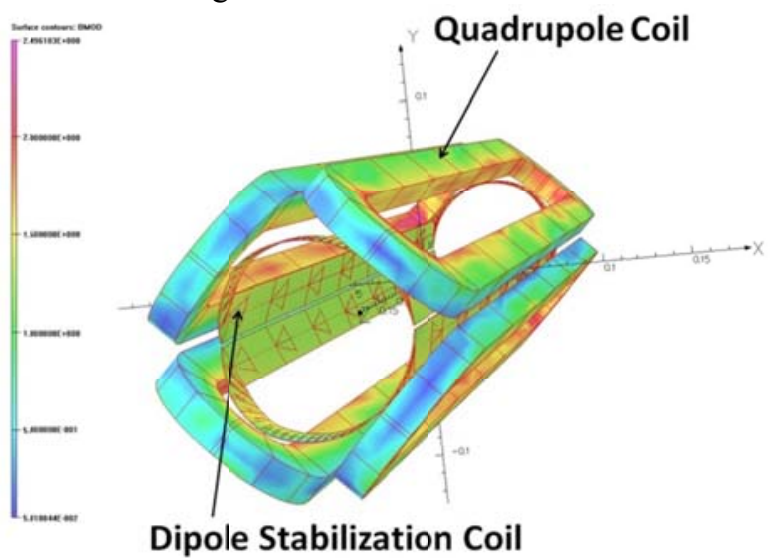

Fig. 5. Quadrupole model with dipole stabilization coils.

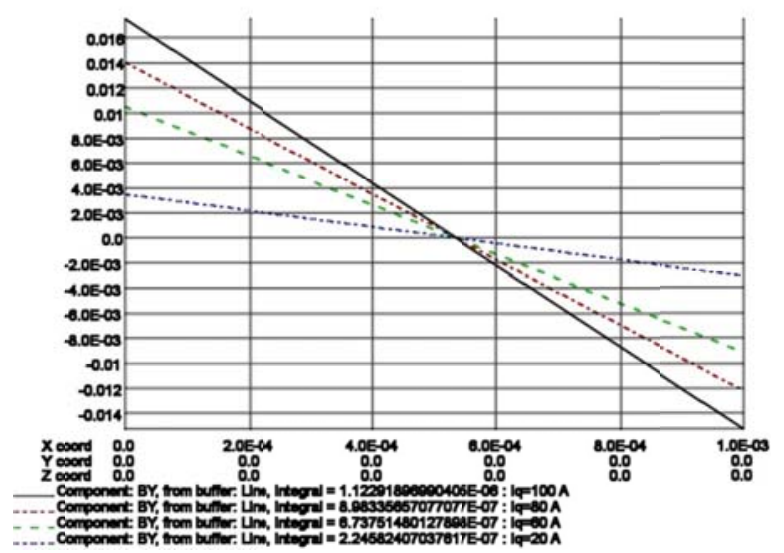

Fig. 6. Quadrupole middle plane magnetic field variations at different currents near by the quadrupole magnetic center.

The Fig. 6 shows that the quadrupole magnetic center is very stable at quadrupole currents $20 \div 100 \mathrm{~A}$. The dipole winding consists of two shell type coils having 74 turns each. In this coils at $1 \mathrm{~mm}$ dipole center shift relatively the quadrupole 
winding at $100 \mathrm{~A}$ in the quadrupole was induced stabilization current $-16.7 \mathrm{~A}$. In the real magnet the specified tolerance is $0.3 \mathrm{~mm}$. Because the induced current is proportional to the quadrupole flux coupled with the dipole winding and depends as a square of coil displacement, the induced current will be less than $1.7 \mathrm{~A}$.

There were investigated also variants of magnet with the iron core having shell (See Fig. 5) or racetrack (See Fig. 7) dipole stabilization coils.

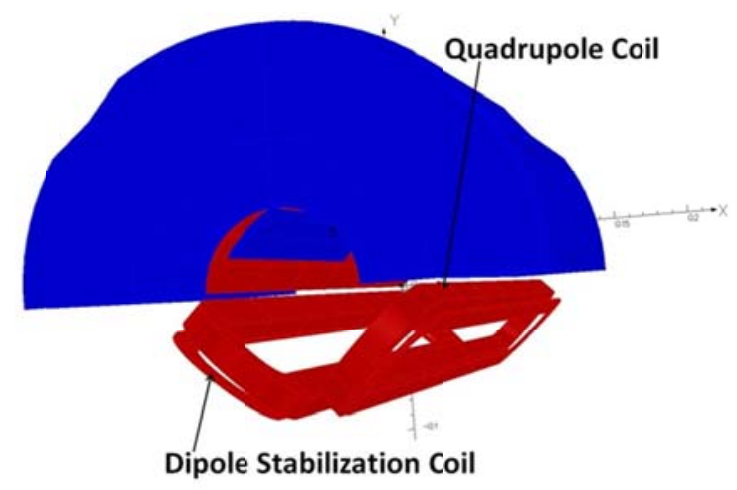

Fig. 7. Quadrupole model with stabilization coils.

The dipole racetrack coils were shifted for $2 \mathrm{~mm}$ in the Xdirection, and three degrease rotated around $\mathrm{Z}$.

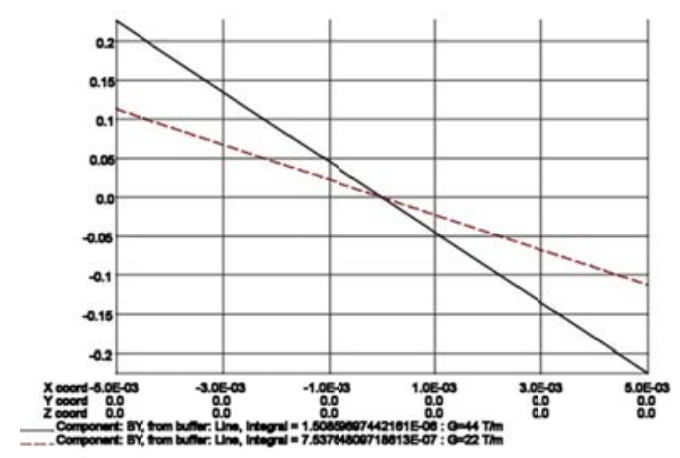

Fig. 8. Quadrupole middle plane magnetic field variations at different field gradients near by the quadrupole magnetic center.

Shown in Fig. 8 field distribution in the case of racetrack coils combined with quadrupoles is less sensitive to the coil position variations.

\section{Cost SAVINGS}

The implementation of the proposed technique for Linear Accelerators may substantially reduce the magnet system cost. In this case, a large number of the following components are eliminated:

- Power supplies;

- Current leads;

- Quench detection system;

- External quench protection system with heater firing units.

The magnet system performance is improved:

- High magnetic center stability provided by stabilization dipole coils;
- Zero noise from power supplies during operation;

- Zero fringing magnetic fields from leads, and buses;

- High reliability passive quench protection system without external detection and protection systems.

Besides, in this case, the magnet specification may be more relaxed to the magnet design, and a fabrication technology.

\section{CONCLUSION}

The proposed approach could be efficient for Superconducting Linear Accelerators. The magnet system in this case will be substantially simplified, more robust, and reliable. The persistent mode of operation, stabilization coil performance will be verified by testing the existing quadrupole model combined with previously tested superconducting switches.

\section{ACKNOWLEDGMENT}

The author would like to thank N. Andreev, V.V. Kashikhin for useful discussions, and D. Turrioni, E. Barzi for the persistent current switch test.

\section{REFERENCES}

[1] "International Linear Collider Reference Design Report," http://www.linearcollider.org/cms/?pid=1000025.

[2] "TESLA Design Report," Hamburg, DESY 2001-11, 2001.

[3] "Fermilab New Muon Laboratory," 2011, http://ilc.fnal.gov/accelerators/new muon.html

[4] "Fermilab Project-X," Document Database, 2011, http://projectx-docdb.fnal.gov/cgi-bin/DocumentDatabase/

[5] V. S. Kashikhin et al., "Design and Manufacturing Main Linac Superconducting Quadrupole for ILC at FERMILAB," IEEE Transactions on Applied Superconductivity, vol. 18, No. 2, June 2008, pp. $155-158$.

[6] A. Koski, R. Bandelmann, S. Wolff, "Superconducting magnet package for the TESLA test facility," IEEE Transactions on Magnetics, vol. 32, No. 4, July 1996, pp. 2155-2158.

[7] F. Toral et al., "Design and fabrication study on the TESLA500 superconducting magnets," IEEE Transactions on Applied Superconductivity, vol. 12, No. 1, March 2002, pp. 282-286.

[8] V.S. Kashikhin, et al., "Superconducting Magnets for SCRF Cryomodules at Front End of Linear Accelerators," Proceedings of IPAC'10, Kyoto, Japan, 2010, pp. 379- 381.

[9] F. Toral,_et. al., "Magnetization Effects on the Superconducting Combined Magnet Prototype for XFEL," IEEE Transactions on Applied Superconductivity, vol. 19. No. 3, June 2009, pp. 1136-1140.

[10] V. S. Kashikhin, et. al., "Test Results of a Superconducting Quadrupole Model Designed for Linear Accelerator Applications," IEEE Transactions on Applied Superconductivity, vol. 19. Issue 3, Part 2, June 2009, pp. 1176-1181.

[11] M.N. Wilson, "Superconducting Magnets," Oxford University Press, Oxford, 1983, p. 330.

[12] T.K. Ko, Y.S. Oh, S.J. Lee, "Optimal Design of the Superconducting Persistent Current Switch with respect to the Heater Currents \& Operating Currents," IEEE Transactions on Applied Superconductivity, vol. 5. No. 2, June 1995, pp. 262- 265.

[13] S.D. Peck, J.C. Zeigler, "A Fast Persistent Switch for microSMES," IEEE Transactions on Applied Superconductivity, vol. 9. No. 2, June 1999, pp. 177- 180.

[14] Rock-Kil KO, et al., "Performance Test of a Persistent Current Switch Insulated with Cotton Tape," IEEE Transactions on Applied Superconductivity, vol. 11. No. 1, June 2001, pp. 2425- 2428.

[15] D. Turrioni, "Test Results of Persistent Current Switches for Linear Accelerators (Unpublished work style)," unpublished.

[16] Y. Tsuda, et al., "Development of Superconducting Mechanical Persistent Current Switch," IEEE Transactions on Applied Superconductivity, vol. 10. No. 1, March 2000, pp. 804- 807. 